\title{
Dynamic Multiaircraft Cooperative Suppression Interference Array Optimization by Dynamic MOPSO Algorithm
}

\author{
Huan Zhang $\mathbb{D}^{1},{ }^{1}$ Rennong Yang $\mathbb{D},{ }^{1}$ and Changyue Sun ${ }^{2}$ \\ ${ }^{1}$ Air Force Engineering University, Xian 710038, China \\ ${ }^{2}$ Tianjin University, Tianjin 300072, China \\ Correspondence should be addressed to Huan Zhang; 315352085@qq.com
}

Received 31 March 2018; Revised 10 June 2018; Accepted 12 June 2018; Published 16 July 2018

Academic Editor: Eduardo Rodriguez-Tello

Copyright (c) 2018 Huan Zhang et al. This is an open access article distributed under the Creative Commons Attribution License, which permits unrestricted use, distribution, and reproduction in any medium, provided the original work is properly cited.

\begin{abstract}
Dynamic multiaircraft cooperative suppression interference array (MACSIA) optimization problem is a typical dynamic multiobjective optimization problem. In this paper, the sum of the distance between each jamming aircraft and the enemy air defense radar network center and the minimum width of the safety area for route planning are taken as the objective functions. The dynamic changes in the battlefield environment are reduced to two cases. One is that the location of the enemy air defense radar is mobile, but the number remains the same. The other is that the number of the enemy air defense radars is variable, but the original location remains unchanged. Thus, two dynamic multiobjective optimization models of dynamic MACSIA are constructed. The dynamic multiobjective particle swarm optimization algorithm is used to solve the two models, respectively. The optimal dynamic MACSIA schemes which satisfy the limitation of the given suppression interference effect and ensure the safety of the jamming aircraft themselves are obtained by simulation experiments. And then verify the correctness of the constructed dynamic multiobjective optimization model, as well as the feasibility and effectiveness of the dynamic multiobjective particle swarm optimization algorithm in solving dynamic MACSIA problem.
\end{abstract}

\section{Introduction}

Under the conditions of high-tech warfare, the air defense radar network on the enemy air defense position usually has a strong ability to resist 'the four', that is, antilow altitude intrusion, antijamming, antistealth, and antiradiation missile $[1,2]$. It is usually difficult for combat aircraft to try to break through the enemy air defense radar network position safely. At this time the combat aircraft must rely on multiple jamming aircraft to implement cooperative suppression interference against the enemy air defense radar network. So that it can provide a secure and planned space for the follow-up route planning of the combat aircraft. In order to make more rational use of limited interference resources, a reasonable array mode should be considered for the location of each jamming aircraft in the process of cooperative interference. Since the actual battlefield environment is constantly changing, it must be considered how to adjust the location of each jamming aircraft based on the actual situation to achieve the best array mode when the battlefield changes. And this problem is the dynamic MACSIA optimization problem in electronic warfare mission planning.

For this problem, in [3], a multiconstraint genetic algorithm is used to solve the deploy optimization problem of the enemy radar network. In [4], Ruan et al. analyzed the various factors that affect the effect of suppression interference and selected the minimum interference distance as a measure for the effect of suppression interference. A weighted sum method was adopted for constructing a single-objective MACSIA model to study the optimal electronic warfare array against radar network [5]. However, the shortcoming of the single-objective optimization model is obvious. Firstly, the determination of the weight depends on the relative importance of each subobjective. Secondly, the experimenter needs to set different weights by multiple experiments to obtain different solutions and the computational overhead is larger. In a work by Zhang et al. [6], a multiobjective optimization model of MACSIA was constructed under static conditions. A modified multiobjective particle swarm optimization algorithm is used to solve the static MACSIA 
optimization problem [7]. Literatures 6 and 7 are our previous research results, but only the MACSIA optimization problem under static conditions is studied. The MACSIA optimization problem is not solved as a dynamic multiobjective optimization problem $[8,9]$ and the impact of changes in the battlefield environment is ignored.

In view of the above problems, in this paper, from the perspective of the real battlefield environment, the dynamic changes in the battlefield environment are reduced to two cases. One is that the location of the enemy air defense radar is mobile, but the number remains the same. The other is that the number of the enemy air defense radars is variable, but the original location remains unchanged. As a basis, on the basis of fully considering the dynamic multiobjective optimization characteristics of the dynamic MACSIA, the sum of the distance between each jamming aircraft and the enemy air defense radar network center and the minimum width of the safety area for route planning are taken as the objective functions. So two dynamic multiobjective optimization models of dynamic MACSIA are constructed. Dynamic multiobjective particle swarm optimization (DMOPSO) [10-18] algorithm has a better effect on the optimization of dynamic environment. It can obtain the optimal solution quickly and effectively. As the algorithm is simple, so the implementation of the project is not difficult and it is widely applied in dynamic multiobjective optimization field [19-22]. Therefore, this paper uses DMOPSO algorithm to solve the two dynamic multiobjective optimization models of dynamic MACSIA. The optimal dynamic MACSIA schemes of each jamming aircraft against the enemy air defense network are calculated, which can provide some reference for the decision-maker to make the next decision.

The novelty of the paper is mainly reflected in the following aspects.

Firstly, to the best of our knowledge, this is the first work that deals with the MACSIA optimization problem as a dynamic multiobjective optimization problem. However, the previous work has considered the problem as a singleobjective optimization or static multiobjective optimization problem to solve. The impact of changes in the battlefield environment was ignored.

Secondly, this paper starts with the characteristics of the real battlefield environment and considers two different environmental changes. One is that the location of the enemy air defense radar is mobile, but the number remains the same. The other is that the number of the enemy air defense radars is variable, but the original location remains unchanged. Thus, two dynamic multiobjective optimization models of dynamic MACSIA are constructed.

Thirdly, the DMOPSO algorithm is used to solve these two models, and the results obtained meet the requirements of the mission. At the same time, the correctness of the model is verified and the feasibility of the DMOPSO algorithm to solve the problem is verified.

Finally, when the battlefield environment changes, it can provide a basis for decision-maker to make decisions. Therefore, the research on the dynamic MACSIA optimization problem has more practical significance.

\section{Model Constructing}

2.1. Dynamic Multiobjective Optimization Model of Dynamic MACSIA. A typical maximized dynamic multiobjective optimization problem can be described as the following mathematical expression $[23,24]$.

$$
\begin{array}{ll}
\max & f(x, t)=\left\{f_{1}(x, t), f_{2}(x, t), \cdots, f_{m}(x, t)\right\} \\
\text { s.t. } & g(x, t)>0, \\
& h(x, t)=0
\end{array}
$$

In this equation, $f(x, t)$ is the set of objective functions with the time variable $t, x \in\left[x_{1}, x_{2}, \cdots, x_{\text {Dim }}\right]^{T}$ represents the decision variable, Dim is the number of decision variables, $m$ is the number of objective functions, and $g(x, t)$ and $h(x, t)$ represent the constraint functions with the time variable $t$.

The ultimate goal of dynamic MACSIA optimization is to achieve a reasonable array of jamming aircraft when the battlefield environment changes, which achieve the best effect of suppression interference against the enemy radar network and ensure the safety of the jamming aircraft themselves. So we can get two dynamic multiobjective optimization models of dynamic MACSIA under two kinds of battlefield environment are as follows.

The first case: the location of the enemy air defense radar is mobile, but the number remains the same.

$$
\begin{aligned}
& f_{1}=\sum_{i=1}^{N_{E C M}}\left(\left(x_{i}-x_{t c}(t)\right)^{2}+\left(y_{i}-y_{t c}(t)\right)^{2}\right. \\
& \left.+\left(z_{i}-z_{t c}(t)\right)^{2}\right)^{1 / 2} \\
& f_{2}=\text { Width }_{\text {safe }} \\
& \text { s.t. } \operatorname{sqrt}\left(\left(x_{i}-x_{t j}(t)\right)^{2}+\left(y_{i}-y_{t j}(t)\right)^{2}+\left(z_{i}\right.\right. \\
& \left.\left.-z_{t j}(t)\right)^{2}\right)>R_{j} \\
& i=1,2, \cdots, N_{E C M} ; j=1,2, \cdots, N_{\text {radar }} \\
& z_{\min } \leq z_{i} \leq z_{\max }
\end{aligned}
$$

Here, the objective function $f_{2}$ represents the minimum width of the safety area for route planning, and the physical meaning is the suppression interference effect of jamming aircraft against the enemy radar network. The objective function $f_{1}$ represents the sum of the distance between each jamming aircraft and the enemy air defense radar network center, and the physical meaning characterizes the safety of jamming aircraft themselves. $\left(x_{t c}(t), y_{t c}(t), z_{t c}(t)\right)$ indicates the coordinate of the enemy air defense radar center; $\left(x_{i}, y_{i}, z_{i}\right)$ indicates the coordinate of the $\mathrm{i}$-th jamming aircraft. In the first case, the location of the enemy air defense radar is mobile, so the coordinate of the enemy air defense radar network is variable and the function of time variable t. Width $h_{\text {safe }}$ is the minimum width of route planning safety area. In the constraints, the location of each jamming aircraft should be outside the maximum detection range of each 
enemy air defense radar, the height of each jamming aircraft should be within the required safety altitude range, and $z_{\max }$ and $z_{\min }$ represent the maximum and minimum values for a given height, respectively. $N_{E C M}$ indicates the number of jamming aircraft, $\left(x_{t j}(t), x_{t j}(t), x_{t j}(t)\right)$ is the coordinate of the i-th enemy air defense radar, and the function of time variable $t . R_{j}$ indicates the maximum detection range of the $i$-th enemy air defense radar; $N_{\text {radar }}$ represents the number of radars in the enemy air defense radar network.

The second case: the number of the enemy air defense radar is variable, but the original location remains unchanged.

In the second case, as the number of the enemy air defense radars is variable, so the number of our jamming aircraft will change accordingly and the location of the enemy air defense radar network center is also changing.

$$
\begin{aligned}
& \begin{aligned}
f_{1} & =\sum_{i=1}^{N_{E C M}(t)}\left(\left(x_{i}-x_{t c}(t)\right)^{2}+\left(y_{i}-y_{t c}(t)\right)^{2}\right. \\
& \left.+\left(z_{i}-z_{t c}(t)\right)^{2}\right)^{1 / 2}
\end{aligned} \\
& f_{2}=\text { Width }_{\text {safe }} \\
& \text { s.t. } \operatorname{sqrt}\left(\left(x_{i}-x_{t j}(t)\right)^{2}+\left(y_{i}-y_{t j}(t)\right)^{2}+\left(z_{i}\right.\right. \\
& \left.\left.\quad-z_{t j}(t)\right)^{2}\right)>R_{j} \\
& \quad i=1,2, \cdots, N_{E C M}(t) ; j=1,2, \cdots, N_{\text {radar }}(t) \\
& z_{\min } \leq z_{i} \leq z_{\max }
\end{aligned}
$$

In this equation, $N_{E C M}(t)$ indicates the number of jamming aircraft and is the function of time variable $t . N_{\text {radar }}(t)$ represents the number of radars in the enemy air defense radar network and is the function of time variable $t$. The meaning of the remaining parameters is the same as in equation (1) .

2.2. Calculation of Route Planning Safety Area Width. The definition of route planning safety area refers to a range of width and height of the combat aircraft within the flight path space and constitutes the follow-up optimal route planning search space for the combat aircraft. In order to facilitate the study, this paper still mainly considers the calculation of route planning safe area width in a certain height of the scope for the combat aircraft. And the width refers to the minimum width of the entire route planning safety area. The method of solving the width of the safe area is still based on the mathematical morphology [25] method. Due to the detection boundary shape of the detection range of the enemy air defense radar network is irregular under terrain shading and electronic jamming conditions, so the traditional geometric method is difficult to calculate the safety area width. The core of mathematical morphology is that you can use a probe structure element to detect the image and obtain the relevant information, so as to achieve the purpose of image analysis and processing. Firstly, the images of the enemy air

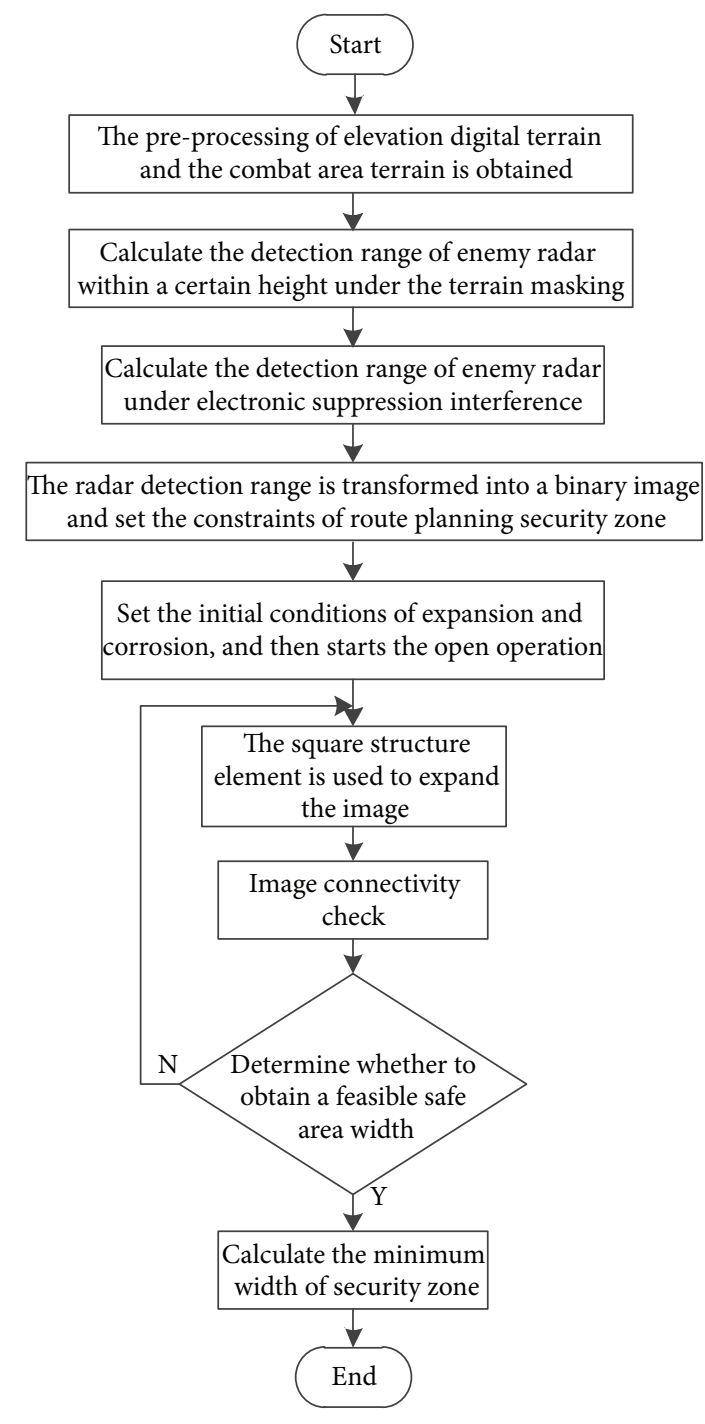

FIGURE 1: The minimum width calculation process of route planning safety area.

defense radar network are binarized according to the terrain shelter and the electronic jamming conditions. Secondly, using the principle of graphic compression and expansion in mathematical morphology, the binary image of the radar detection range is opened and then the expansion operation is carried out, and the image connectivity is continuously checked. Finally, determine whether the minimum width of the route planning safety zone satisfying the given width limit is formed and the minimum width is calculated. On this part of the content which can also be seen in our previous research results $[6,7]$, the specific calculating steps are shown in Figure 1.

The calculation model of the range of enemy air defense radar under terrain shading and electronic jamming in the process is as follows $[6,7]$.

The detection range calculation of the enemy air defense radar under terrain shading is as follows.

Figure 2 shows the detection range calculation diagram of the enemy air defense radar under terrain shading. 


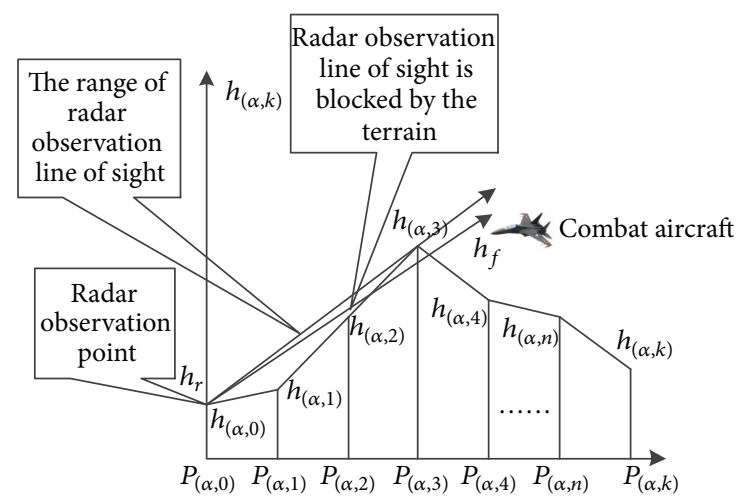

FIGURE 2: The detection range calculation diagram of the enemy air defense radar under terrain shading.

In Figure 2, the combat aircraft is the target point, the height of which is expressed by $h_{f}$. In $\alpha$ direction, the farthest boundary point of the enemy radar is represented by the polar coordinate grid $P_{(\alpha, 3)}$, where the elevation value is denoted by $h_{(\alpha, 3)} \cdot h_{r}$ represents the height of radar observation point. The height angle $\varepsilon_{0}$ from the enemy radar observation point to the target and the height angle $\varepsilon_{P_{(\alpha, k)}}$ from the enemy radar observation to $P_{(\alpha, k)}$ can be obtained in Figure 2, respectively. As shown in (4), calculate $\varepsilon_{P_{(\alpha, k)}}(k=0,1,2 \cdots k)$ in turn and compare with $\varepsilon_{o}$, respectively. If there is a $P_{(\alpha, k)}$ value satisfying $\varepsilon_{P_{(\alpha, k)}}>\varepsilon_{o}$, then the enemy radar observation line of sight is blocked by the terrain and the target can not be detected.

$$
\begin{aligned}
\varepsilon_{P_{(\alpha, k)}} & =\arctan \frac{h_{(\alpha, k)}-h_{r}}{P_{(\alpha, k)}-P_{(\alpha, 0)}} \\
\varepsilon_{o} & =\arctan \frac{h_{f}-h_{r}}{P_{(\alpha, n)}-P_{(\alpha, 0)}}
\end{aligned}
$$

The detection range calculation of the enemy air defense radar under electronic jamming is as follows.

The detection range expression of multiple jamming aircraft cooperative suppression interference against the enemy air defense radar network is shown in

$$
R=\left(\frac{P_{t} G^{2} \sigma \lambda^{2}}{(4 \pi)^{3} L F_{n} k T_{o} B_{n} K_{j}+\sum_{i=1}^{n}\left(4 \pi L P_{j i} G_{j i} G\left(\varphi_{i}\right) r_{j i} B_{r} / R_{j i}^{2} L_{j i} B_{j i}\right) K_{j}}\right)^{1 / 4}
$$

Here, $R_{j i}$ is the distance between the enemy radar and the i-th jamming aircraft and its unit is $\mathrm{m}, L_{j i}$ represents the interference loss of the i-th jamming aircraft, $B_{j i}$ indicates the interference signal bandwidth of the i-th jamming aircraft and its unit is $\mathrm{Hz}$, and $P_{j i}$ is the transmit power of the $\mathrm{i}$-th jamming aircraft and its unit is W. $G_{j i}$ represents the antenna gain of the i-th jamming aircraft, $r_{j i}$ indicates the polarization loss of the i-th jamming aircraft, $B_{r}$ represents the signal bandwidth received by the enemy radar receiver and its unit is $\mathrm{Hz}, L$ is the system loss of the enemy radar, $B_{n}$ indicates the noise bandwidth of the enemy radar, $T_{0}$ is the current working environment temperature of the enemy radar and its unit is $K, k$ represents the Boltzmann constant which is $1.38 \times 10^{-23} \mathrm{~J} / \mathrm{K}, F_{n}$ indicates the noise coefficient of the enemy radar, $\lambda$ represents the wavelength of the enemy radar and its unit is $\mathrm{m}, \sigma$ indicates the target cross-sectional area of the enemy radar and its unit is $\mathrm{m}^{2}, G$ is the antenna gain of the enemy radar, $P_{t}$ represents the emission peak power of the enemy radar and its unit is $\mathrm{W}$, and $n$ indicates the number of the jamming aircraft. When $n$ is 1 , formula (5) shows the detection distance of single jamming aircraft suppression interference against the enemy radar. $K_{j}$ is the minimum suppression factor of the enemy radar, $G\left(\varphi_{i}\right)$ represents the gain of the enemy radar antenna in the interference direction of the $\mathrm{i}$-th jamming aircraft, and the expression is shown in

$$
G(\varphi)= \begin{cases}G, & 0 \leq|\varphi| \leq \varphi_{0.5} \\ K\left(2\left(\frac{\varphi_{0.5}}{\varphi}\right)\right)^{2} G, & \varphi_{0.5} \leq|\varphi| \leq 90^{\circ} \\ K\left(2\left(\frac{\varphi_{0.5}}{90^{\circ}}\right)\right)^{2} G, & 90^{\circ} \leq|\varphi| \leq 180^{\circ}\end{cases}
$$

In the above equation, the value range of $K$ is generally 0.04 to 0.1 and $\varphi_{0.5}$ indicates the lobe width of the enemy radar antenna at the half power point.

\section{Dynamic Multiobjective Particle Swarm Optimization Algorithm}

3.1. Particle Swarm Optimization. Particle swarm optimization (PSO) [26] algorithm is widely used in solving single target optimization problem. The updating location and speed expressions of particles in PSO are shown in

$$
\begin{aligned}
X_{i+1} & =X_{i}+V_{i+1} \\
V_{i+1} & =w V_{i}+c_{1} r_{1}\left(\text { Pbest }_{i}-X_{i}\right)+c_{2} r_{2}\left(\text { Gbest }_{i}-X_{i}\right)
\end{aligned}
$$

In this equation, $V_{i}$ indicates the current speed of the particle, $V_{i+1}$ represents the speed at the next moment, $X_{i}$ indicates the current location of the particle, the position of the particle at the next moment is denoted by $X_{i+1}$, Gbest ${ }_{i}$ is the global optimal solution for the i-th generation of population, Pbest $_{i}$ represents the individual optimal solution for the ith generation of particle, and $r_{1}$ and $r_{2}$ are random numbers with values between 0 and 1 . Both $c_{1}$ and $c_{2}$ represent learning factors, $w$ indicates the inertia weight factor, which is a variable that reflects the effect of the current velocity of the particle on the velocity of the particle at the next moment, and the expression is

$$
w=w_{\max }-\frac{w_{\max }-w_{\min }}{\text { iter }_{\max }} * \text { itera }
$$


Here, itera is the current number of population iterations, itera $_{\max }$ represents the maximum number of population iterations, and $w_{\min }$ and $w_{\max }$ represent the minimum and maximum values of the inertia weighting factor, respectively.

3.2. DMOPSO Algorithm. DMOPSO algorithm is to optimize the function with multiple objective functions in the dynamic environment, while optimizing the objective functions to achieve the best condition. In the dynamic environment, the optimal solution of the individual and the optimal solution of the population may change over time, and the particle is easy to fall into the optimization state of the former environment and stagnate. Therefore, the core of the dynamic multiobjective optimization problem is to adapt to environmental changes and can detect changes in the environment quickly and accurately and how to make adjustments to adapt to environmental changes. In this paper, the dynamic environment monitoring method based on sentinel particles [20] is used. The basic idea of this method is to generate a part of the sentinel particles randomly as the population is initialized at the same time. The fitness values of the sentinel particles are calculated in each iteration. When the fitness values of the sentinel particles change, it is determined that the current environment has changed. The sentinel particles do not participate in each iteration, so the fitness values of the sentinel particles should remain constant in the static environment. When monitoring the environment changes, the DMOPSO algorithm needs to adjust the current optimization results. Firstly, the information in the nondominated solution memory is recalculated under the current environmental conditions, the dominated solution is removed, and then a part of the particle location in the search space is reinitialized. Generally, thirty percent of the particles are selected. The specific flow chart of the dynamic MOPSO algorithm based on sentinel particle monitoring is depicted in Figure 3.

Based on the process of DMOPSO algorithm, formulae (1) and (3) are used as the objective functions, and then the calculation method of the route planning safety area minimum width in Section 3 is combined. So the dynamic MACSIA array schemes can be obtained in the case of two kinds of battlefield environment dynamic changes, which satisfy the limitation of the given suppression interference effect and ensure the safety of the jamming aircraft themselves.

\section{Simulation Experiment}

Simulation environment is in Windows 7 32-bit system, and the processor is the Intel (R) Core (TM) i5-4590 CPU @ 3.3 $\mathrm{GHz}$. The programming language is programmed with MATLAB 2010a. A $432 \mathrm{~km} * 432 \mathrm{~km}$ size area is selected as the simulation experiment of the combat scene. The digital elevation of this region is shown in Figure 4, it uses a grid structure, and the elevation data resolution is $360 \mathrm{~m}$.

The first case: the location of the enemy air defense radar is mobile, but the number remains the same.

Assuming that four enemy air defense radars are deployed in the combat area, the minimum suppression factors $K_{j}$ of

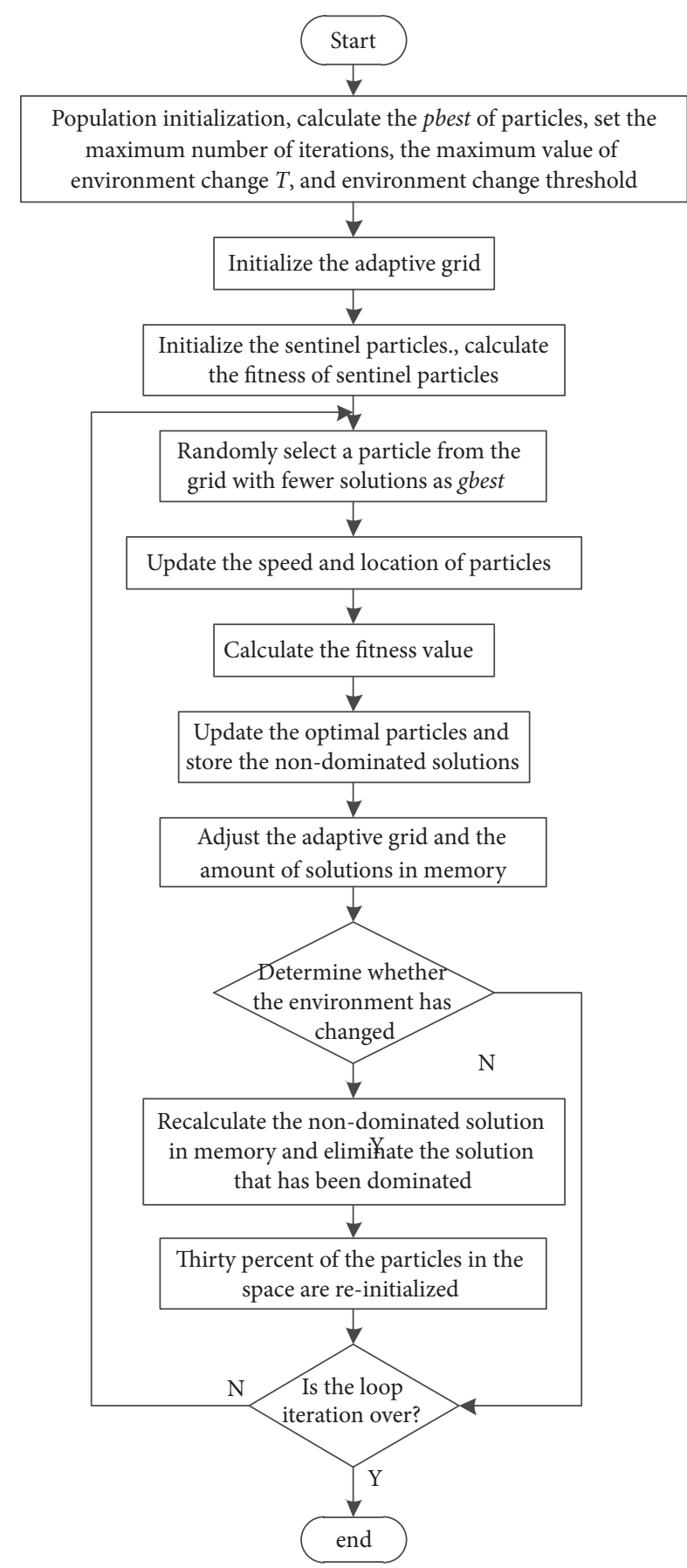

FIGURE 3: The flow chart of DMOPSO algorithm based on sentinel particle monitoring.

the four enemy radars are 5, as shown in Table 1 for the list of performance parameters of the four enemy radars. The initial coordinate locations of the four enemy radars are $(240 \mathrm{~km}, 100 \mathrm{~km}),(150 \mathrm{~km}, 120 \mathrm{~km}),(300 \mathrm{~km}, 250 \mathrm{~km})$, and $(180 \mathrm{~km}, 280 \mathrm{~km})$.

Initially, the detection range of the four enemy air defense radars under terrain shading at a height of $2.1 \mathrm{~km}$ is shown in Figure 5. In the figure, the detection range of the four enemy air defense radars overlaps, so it is very difficult for our 


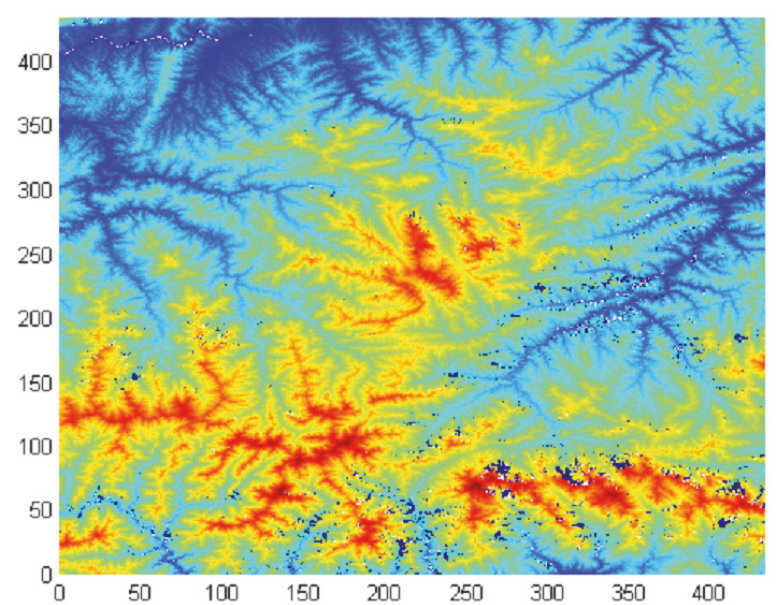

FIgURE 4: Digital elevation terrain map.

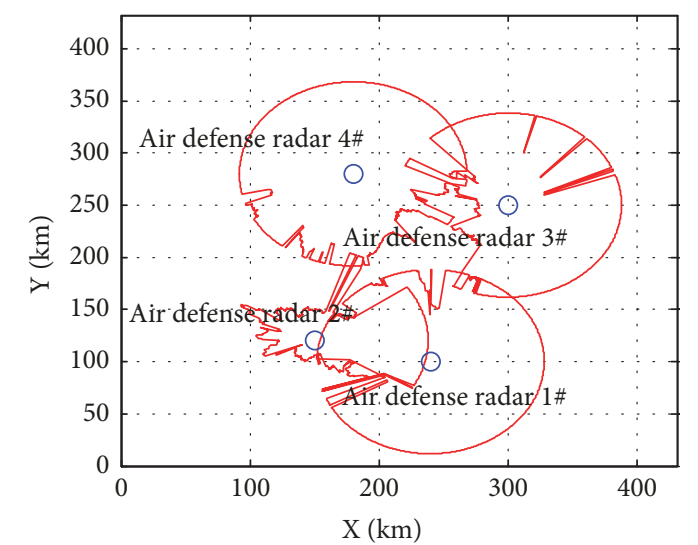

FIgURE 5: The detection range of the four enemy air defense radars under terrain shading at the beginning.

TABLE 1: The list of four enemy air defense radar performance parameters.

\begin{tabular}{lccccccc}
\hline Radar & $P_{t} / \mathrm{kW}$ & $G$ & $\lambda / m$ & $L$ & $F_{n}$ & $T_{o} / K$ & $B_{n} / \mathrm{Hz}$ \\
\hline $1 \#$ & 4000 & 50 & 0.6 & 2.5 & 3 & 291 & $2 \times 10^{5}$ \\
$2 \#$ & 3900 & 45 & 0.8 & 4 & 2.5 & 291 & $2.1 \times 10$ \\
$3 \#$ & 3100 & 70 & 0.75 & 3 & 4 & 291 & $3 \times 10^{5}$ \\
$4 \#$ & 2600 & 100 & 0.5 & 3 & 6 & 291 & $1.5 \times 10$ \\
\hline
\end{tabular}

combat aircraft to penetrate the defense safely. At this time the combat aircraft must rely on the electronic support jamming aircraft to implement cooperative suppression interference against the enemy air defense radar network, so as to obtain a given limit to meet the conditions of the route planning safety area width. The minimum width of the route planning safety area is limited to $20 \mathrm{~km}$. We intend to send three electronic support jamming aircraft to implement cooperative suppression interference against the enemy air defense radar network. The three jamming aircraft are at a height of $2.1 \mathrm{~km}$ and the performance parameters are shown in Table 2.
TABLE 2: The list of three jamming aircrafts parameters.

\begin{tabular}{lccccc}
\hline Jamming aircraft & $P_{j} / k W$ & $G_{j}$ & $r_{j}$ & $L_{j}$ & $B_{j} / H z$ \\
\hline $1 \#$ & $1 \times 10^{3}$ & 4 & 2 & 20 & $2 \times 10^{6}$ \\
2\# & $2 \times 10^{3}$ & 2 & 2 & 20 & $2 \times 10^{6}$ \\
3\# & $2 \times 10^{3}$ & 6 & 1 & 30 & $2 \times 10^{6}$ \\
\hline
\end{tabular}

TABLE 3: The coordinates of the four enemy air defense radars after three environment changes.

\begin{tabular}{lccc}
\hline Radar & $\begin{array}{c}\text { After the first } \\
\text { environment } \\
\text { change }\end{array}$ & $\begin{array}{c}\text { After the Second } \\
\text { environment } \\
\text { change }\end{array}$ & $\begin{array}{c}\text { After the third } \\
\text { environment } \\
\text { change }\end{array}$ \\
\hline $1 \#$ & $(240,100)$ & $(230,100)$ & $(240,105)$ \\
$2 \#$ & $(150,120)$ & $(160,130)$ & $(160,120)$ \\
$3 \#$ & $(300,250)$ & $(300,250)$ & $(300,220)$ \\
$4 \#$ & $(170,270)$ & $(170,270)$ & $(180,250)$ \\
\hline
\end{tabular}

The initial parameters of DMOPSO algorithm are as follows: the population size $x$ Size is 100 , the dimension of decision variables $\operatorname{Dim}$ is 6, the learning factor $c_{1}=c_{2}=0.75$, the maximum and minimum values of the inertia weighting factor are $w_{\max }=1.1, w_{\min }=0.2$, respectively, and the maximum number of iterations Max_itera is 500. The number of environment changes is set to $T=3$, the number of iterations of DMOPSO algorithm is 150 for each time the environment changes, and the threshold of environment change is $\delta=0.5$; set the initial environment $t=0$. As the moving law of the enemy air defense radars is difficult to obtain, therefore, in this paper, for the sake of simplified study, the coordinates (in $\mathrm{km}$ ) of the four enemy air defense radars after three environment changes are shown in Table 3. Here is the detection range of the four air defense radars under terrain shading after the third environment change, as shown in Figure 6.

The experimental results were run independently by DMOPSO algorithm 30 times, and the optimal result was selected from them. The calculated nondominated solutions distribution is shown in Figure 7.

It can be seen from Figure 7 that DMOPSO algorithm has a uniform distribution of nondominated solution sets, which constitute four Pareto front surfaces, and it is concluded that the search effect of DMOPSO algorithm is still good when the environment changes. In this paper, the Pareto front surface generated after the third environmental change is taken as an example. In order to facilitate the decisionmaker to make decisions, three points are selected from the Pareto front surface: the intermediate point $S_{2}$ and the two endpoints $S_{1}$ and $S_{3}$, respectively, where $S_{1}$ is biased to optimize the $f_{2}$ objective and $S_{3}$ is biased towards the optimized $f_{1}$ objective, while $S_{2}$ takes into account both objective $f_{2}$ and $f_{1}$. The optimal dynamic MACSIA schemes of the three points are shown in Figures 8, 9, and 10, respectively.

When the decision-maker who develops the combat plan needs to minimize the sum of the distance between each jamming aircraft and the enemy air defense radar 


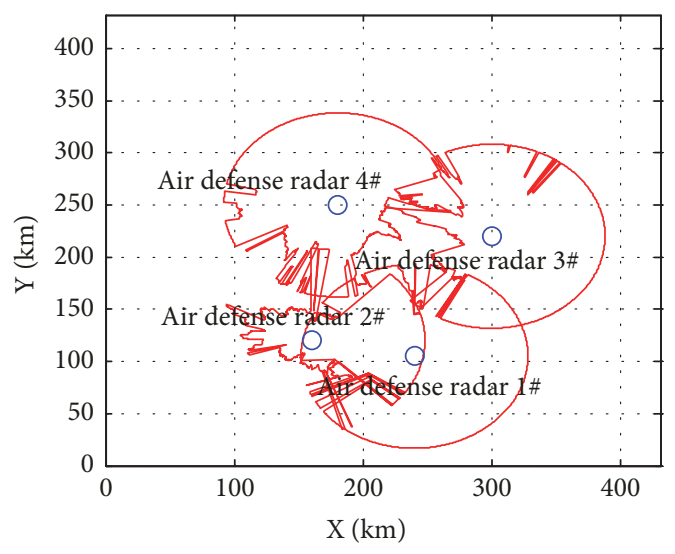

Figure 6: The detection range of the four air defense radars under terrain shading after the third environment change.

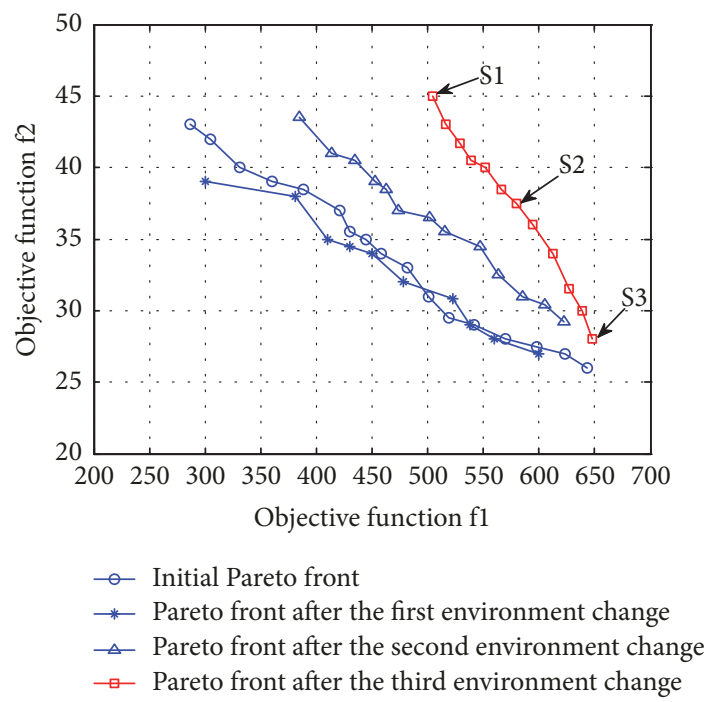

FIGURE 7: The obtained nondominated solutions distribution in the first case.

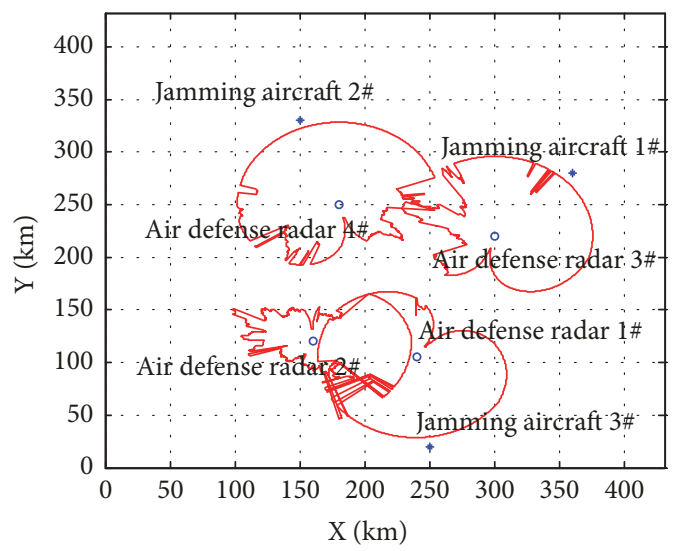

FIgURE 8: The optimal dynamic MACSIA scheme of point $S_{1}$ in the first case.

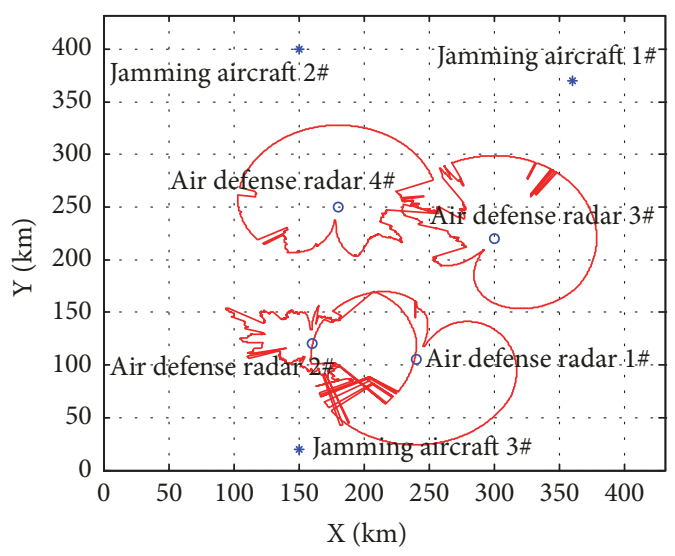

FIgure 9: The optimal dynamic MACSIA scheme of point $S_{3}$ in the first case.

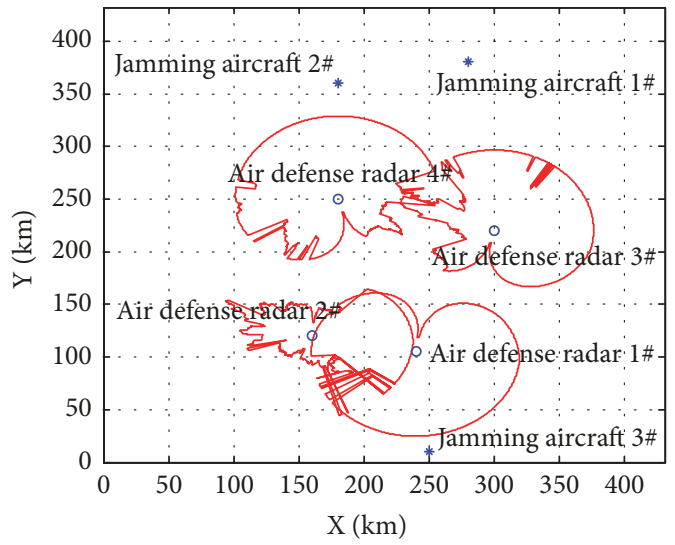

FIgURE 10: The optimal dynamic MACSIA scheme of point $S_{2}$ in the first case.

network center and maximize the minimum width of the route planning safety area, the emphasis is the suppression interference effect on the enemy air defense radar network. And the optimal dynamic MACSIA scheme of point $S_{1}$ can be chosen; as shown in Figure 8, the three jamming aircraft are closer to the enemy air defense radar network center in this scheme. When the decision-maker who develops the combat plan needs to maximize the sum of the distance between each jamming aircraft and the enemy air defense radar network center and minimize the minimum width of the route planning safety area, that is to say, the focus is on the safety of each jamming aircraft itself, the optimal dynamic MACSIA scheme of point $S_{3}$ can be chosen; as shown in Figure 9, the three jamming aircraft are more far away from the enemy air defense radar network center in this scheme. When the decision-maker who develops the combat plan not only needs to consider the safety of each jamming aircraft itself, but also needs to achieve a given suppression interference effect, the optimal dynamic MACSIA scheme of point $S_{2}$ may be the best choice, as it can be seen in Figure 10. At this time the total distance between each jamming aircraft and the enemy air defense radar network center is moderate in this scheme. 


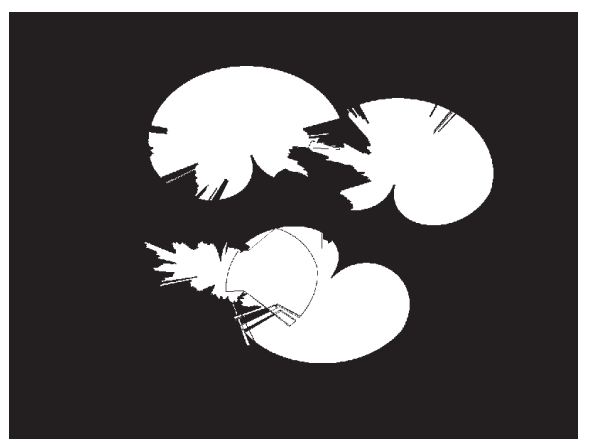

FIGURE 11: The binary image of suppression interference effect of point $S_{1}$ for the jamming aircraft against the enemy air defense radar network in the first case.

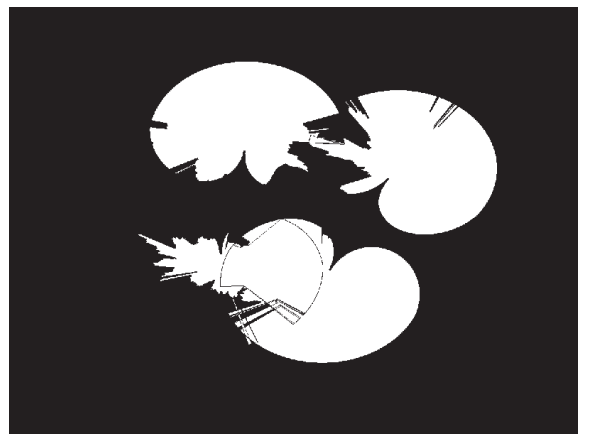

FIGURE 12: The binary image of suppression interference effect of point $S_{3}$ for the jamming aircraft against the enemy air defense radar network in the first case.

Figures 11,12 , and 13 are the binary images of suppression interference effect for the jamming aircraft against the enemy air defense radar network in three schemes. The obtained minimum safety widths are $45 \mathrm{~km}, 28 \mathrm{~km}$, and $37.5 \mathrm{~km}$, respectively.

The second case: the number of the enemy air defense radar is variable, but the original location remains unchanged.

At first, assuming that three enemy air defense radars are deployed in the combat area, the minimum suppression factors $K_{j}$ of the three enemy air defense radars are 5 . The performance parameters are the same as the number 1,2 , and 3 radar in Table 1 . And the initial coordinate locations of the three enemy air defense radars are $(220 \mathrm{~km}, 105 \mathrm{~km})$, $(290 \mathrm{~km}, 230 \mathrm{~km})$, and $(190 \mathrm{~km}, 270 \mathrm{~km})$.

Initially, the detection range of the three enemy air defense radars under terrain shading at a height of $2.1 \mathrm{~km}$ is shown in Figure 14. It can be seen from Figure 13 that the detection range of the three enemy air defense radars overlaps, so it is very difficult for our combat aircraft to penetrate the defense safely. At this time the combat aircraft must rely on the electronic support jamming aircraft to implement cooperative suppression interference against the enemy air defense radar network, so as to obtain a given limit to meet the conditions of the route planning safety area width. The minimum width of the route planning safety

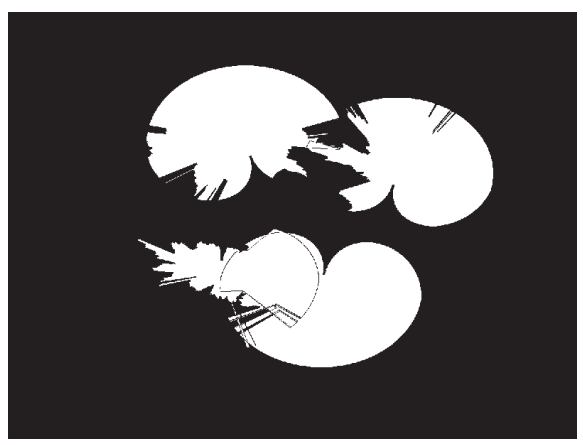

FIGURE 13: The binary image of suppression interference effect of point $S_{2}$ for the jamming aircraft against the enemy air defense radar network in the first case.

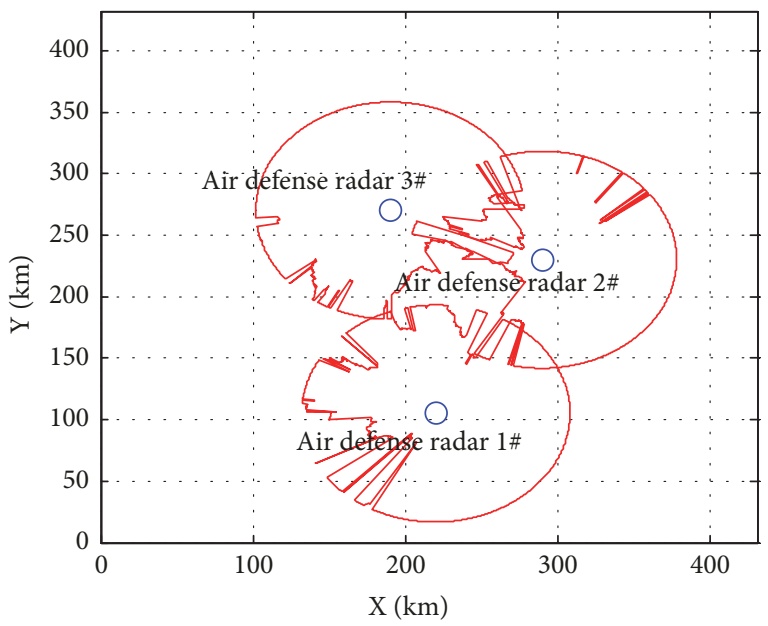

FIGURE 14: The detection range of the three enemy air defense radars under terrain shading at the beginning.

area is limited to $20 \mathrm{~km}$. At the beginning, we intend to send two electronic support jamming aircraft to implement cooperative suppression interference against the enemy air defense radar network. The two jamming aircraft are at a height of $2.1 \mathrm{~km}$ and their performance parameters are the same as the number 1 and 2 jamming aircraft in Table 2.

The initial parameters of DMOPSO algorithm are as follows: the maximum number of iterations Max_itera is 800 , the number of environment changes is set to $T=$ 2 , the number of iterations of DMOPSO algorithm is 250 for each time the environment changes, the threshold of environment change is $\delta=0.6$, and the remaining parameters are consistent with the simulation parameters in the first case. In order to simplify the study, at the same time more in line with the real battlefield environment, the number of enemy air defense radar is limited to 3 to 5 , and the number of enemy air defense radars is increased in the process of environment change. The coordinates of the additional enemy air defense radar after the two environment changes are $(150 \mathrm{~km}, 210 \mathrm{~km})$ and $(300 \mathrm{~km}, 130 \mathrm{~km})$, respectively. Here is given the detection range of the enemy air defense radar network by terrain 


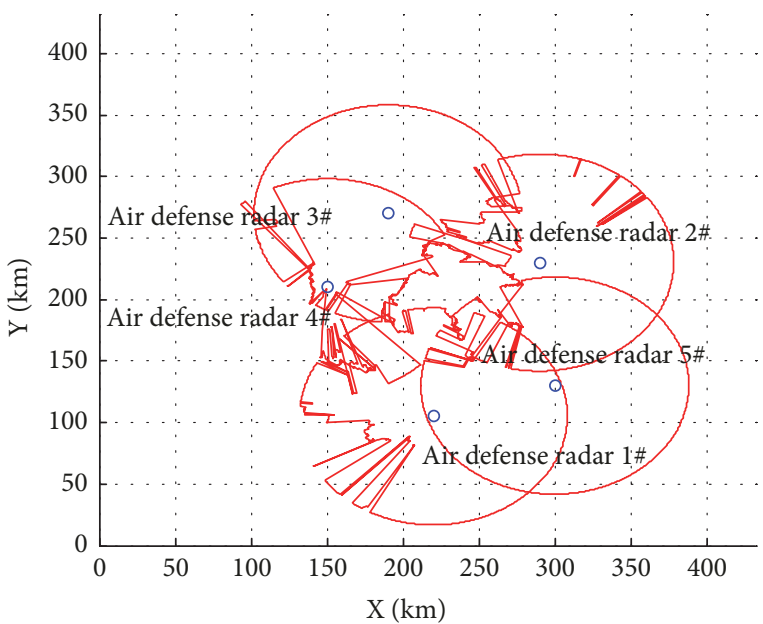

FIGURE 15: The detection range of the five air defense radars under terrain shading after the second environment change.

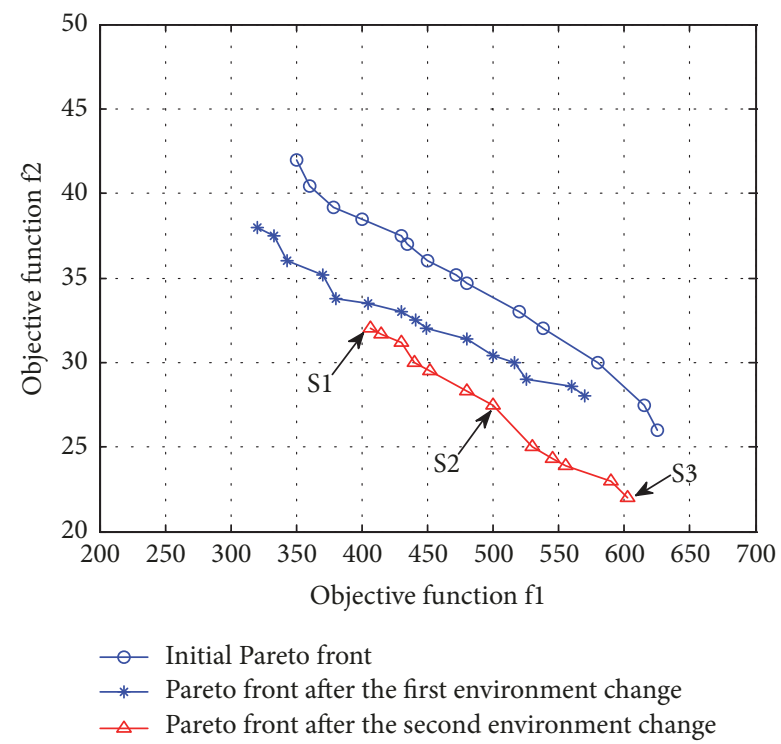

FIGURE 16: The obtained nondominated solutions distribution in the second case.

shading after the second environment change, which is shown in Figure 15.

The experimental results were run independently by DMOPSO algorithm 30 times, and the optimal result was selected from them. The calculated nondominated solutions distribution is shown in Figure 16.

As can be seen from Figure 16, when the environment changes, the search effect of DMOPSO algorithm is still relatively good, and three more uniform Pareto front surfaces are generated. Then the Pareto front surface generated after the second environment change is taken as an example. Similarly, in order to facilitate decision-maker to make decisions, three points called $S_{1}, S_{2}$, and $S_{3}$ are selected on the Pareto frontier surface. The optimal dynamic MACSIA schemes of the three points are shown in Figures 17, 18, and 19, respectively.

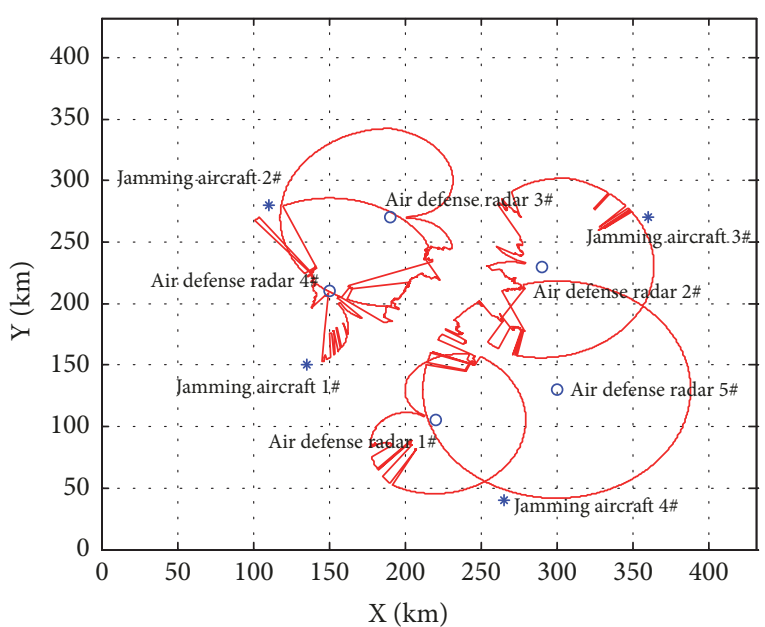

FIgURE 17: The optimal dynamic MACSIA scheme of point $S_{1}$ in the second case.

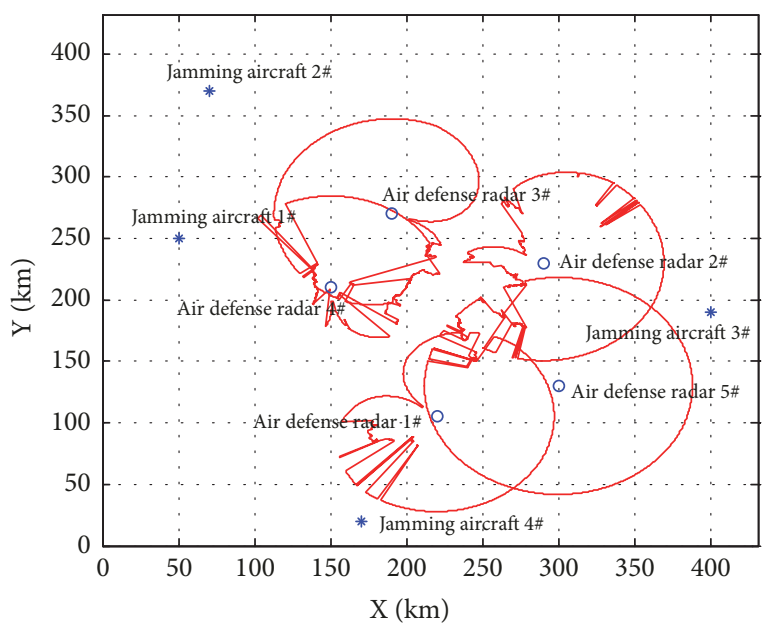

FIGURE 18: The optimal dynamic MACSIA scheme of point $S_{3}$ in the second case.

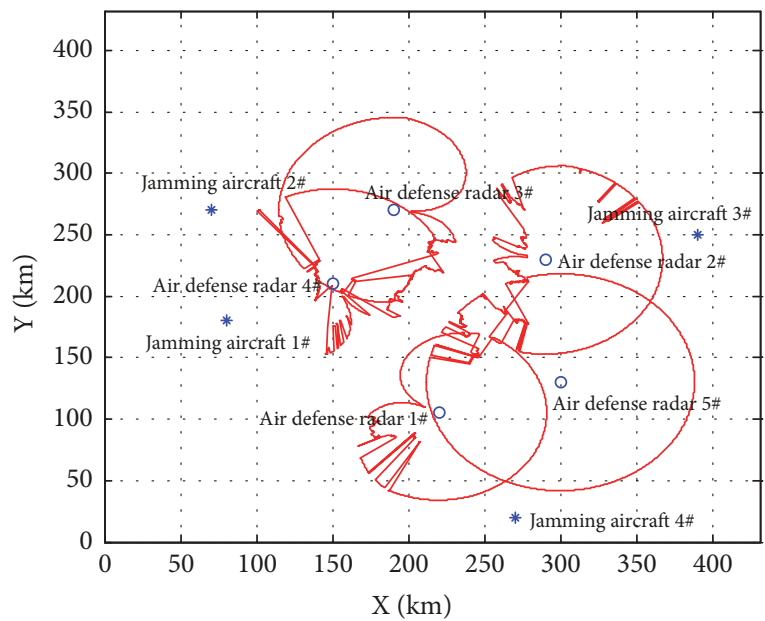

FIgURE 19: The optimal dynamic MACSIA scheme of point $S_{2}$ in the second case. 


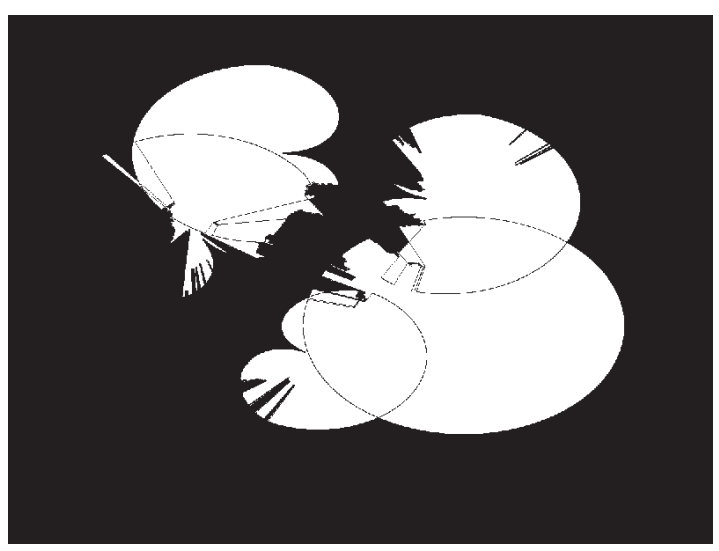

FIGURE 20: The binary image of suppression interference effect of point $S_{1}$ for the jamming aircraft against the enemy air defense radar network in the second case.

As shown in Figure 17, each jamming aircraft is relatively close to the enemy air defense radar network center in this scheme, the total distance is the smallest and the minimum width of the route planning safe area is the largest. The choice of point $S_{1}$ corresponding to the scheme means that more emphasis is on the suppression interference effect on the enemy air defense radar network. Figure 18 shows that the four jamming aircraft are far from the enemy air defense radar network center in this scheme, correspondingly, the sum of the distance between each jamming aircraft and the enemy air defense radar network center is the largest, and the minimum width of the route planning safe area is the smallest. If the focus is on the safety of the jamming aircraft themselves, the point $S_{3}$ corresponding to the scheme can be selected. As shown in Figure 19, at this time, the total distance between the four jamming aircraft and the enemy air defense radar network center is moderate. When the decision-maker who develops the combat plan needs to consider the safety of the jamming aircraft themselves and achieve the given suppression interference effect, the point $S_{2}$ corresponding to the scheme can be selected.

Figures 20,21, and 22 are the binary images of suppression interference effect for the jamming aircraft against the enemy air defense radar network in three schemes. The obtained minimum safety widths are $32 \mathrm{~km}, 22 \mathrm{~km}$, and $27.5 \mathrm{~km}$, respectively.

It can be concluded from the above that the six kinds of dynamic MACSIA schemes have achieved good suppression interference effect in the two cases and formed the route planning safety area which satisfies the restriction condition and ensures the safety of the jamming aircraft themselves. The decision-maker who develops the combat plan can select the corresponding scheme according to the actual operational needs.

\section{Conclusions}

In this paper, aiming at the problem of dynamic MACSIA optimization in electronic warfare mission planning,

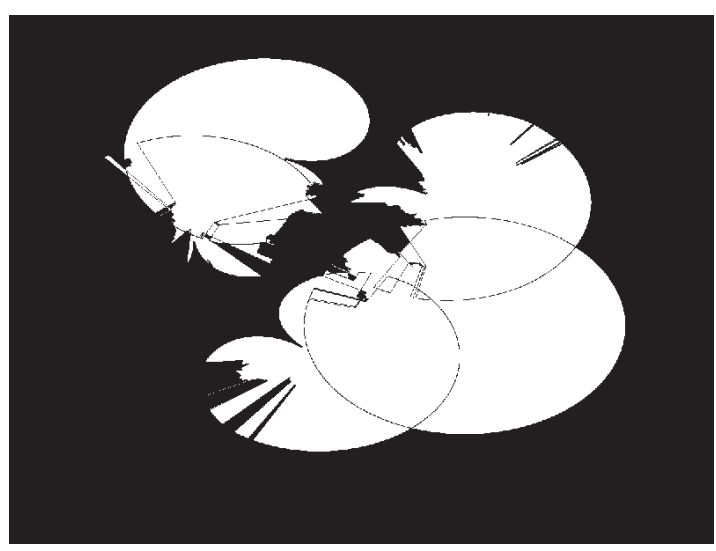

FIGURE 21: The binary image of suppression interference effect of point $S_{3}$ for the jamming aircraft against the enemy air defense radar network in the second case.

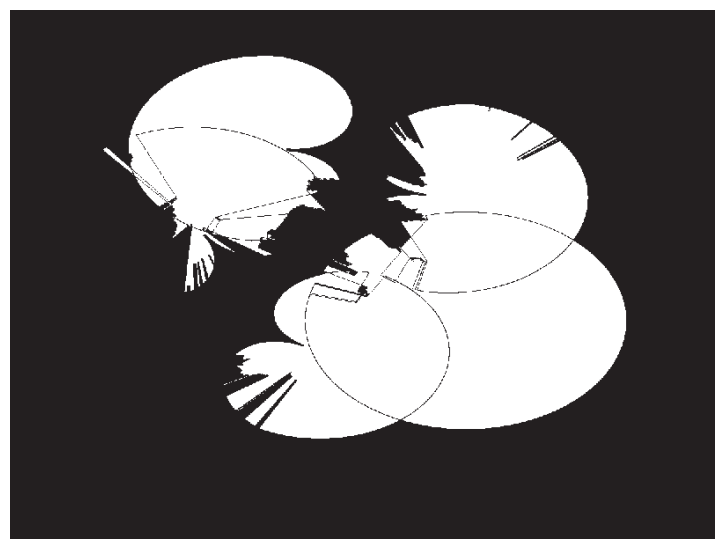

FIGURE 22: The binary image of suppression interference effect of point $S_{2}$ for the jamming aircraft against the enemy air defense radar network in the second case.

based on two different environment changes, two kinds of dynamic multiobjective optimization models of the dynamic MACSIA are constructed by regarding the sum of the distance between each jamming aircraft and the enemy air defense radar network center and the minimum width of the route planning safety area as the objective functions. The DMOPSO algorithm is used to solve the two models, and the optimal dynamic MACSIA schemes are calculated in the case of two environment changes. Simultaneously, the feasibility and validity of the proposed model and the proposed method are verified. The study content of this paper is a key part of the dynamic planning in electronic warfare mission planning. When the battlefield environment changes, it can provide a basis for decision-maker to make decisions and also provide a safe and reliable planning space for the follow-up route planning of the combat aircraft. Therefore, this paper has a relatively strong significance of actual combat. 


\section{Data Availability}

The data used to support the findings of this study are available from the corresponding author upon request.

\section{Conflicts of Interest}

The authors declare that there are no conflicts of interest regarding the publication of this paper.

\section{Authors' Contributions}

Huan Zhang planned the work, completed the simulation experiment, and drafted the main part of the paper. Rennong Yang contributed to error analysis. Changyue Sun contributed to setup type.

\section{Acknowledgments}

The work described in this paper is partially supported by the National Natural Science Foundation of China under Grant no. 61503405 .

\section{References}

[1] X. Zhang, "“Four countering” of radar in the early of the 21st century," Radar Science and Technology, vol. 1, no. 1, pp. 1-6, 2003.

[2] G. L. Fan, Z. M. Yang, and Y. Z. Wang, "Evaluation of four countering efficiency of netted radar based on multi-stage fuzzy synthetic judgment," Shipboard Electronic Countermeasure, vol. 36, no. 3, pp. 100-102, 2013.

[3] Z. Wang, X. Li, Q. Zhou, and W. Wang, "Optimal deployment of radar network based on multi-constrained GA," Systems Engineering and Electronics, vol. 30, no. 2, pp. 265-268, 2008.

[4] M. Z. Ruan, H. J. Wang, and Q. M. Li, "Efficiency evaluation of multi-jamming sources on stand-off jamming based on the exposed range," Journal of Systems Engineering and Electronics, vol. 31, no. 9, pp. 2110-2114, 2009.

[5] Z.-Q. Chen, L. Yu, Y. Lu, and Z.-L. Zhou, "Research on optimized electronic warfare embattling countermining radar net," Acta Armamentarii, vol. 33, no. 1, pp. 89-94, 2012.

[6] H. Zhang, R. Yang, J. Wu, Q. Li, X. Fu, and C. Sun, "Research on multi-aircraft cooperative suppressing jamming embattling in electronic warfare planning," Systems Engineering and Electronics, vol. 39, no. 3, pp. 542-548, 2017.

[7] H. Zhang, R. Yang, C. Sun, and H. Han, "Research on multiaircraft cooperative suppression interference array based on an improved multiobjective particle swarm optimization algorithm," Mathematical Problems in Engineering, vol. 2017, Article ID 9843735, 12 pages, 2017.

[8] S. Biswas, S. Das, P. N. Suganthan, and C. A. C. Coello, "Evolutionary multiobjective optimization in dynamic environments: A set of novel benchmark functions," in Proceedings of the 2014 IEEE Congress on Evolutionary Computation, CEC 2014, pp. 3192-3199, July 2014.

[9] S. Biswas, D. Bose, S. Das, and S. Kundu, "Decompositionbased evolutionary multi-objective optimization approach to the design of concentric circular antenna arrays," Progress in Electromagnetics Research B, no. 52, pp. 185-205, 2013.
[10] M. Helbig and A. P. Engelbrecht, "Analyses of guide update approaches for vector evaluated particle swarm optimisation on dynamic multi-objective optimisation problems," in Proceedings of the 2012 IEEE Congress on Evolutionary Computation, CEC 2012, Australia, June 2012.

[11] C.-A. Liu, "Particle swarm algorithm for solving dynamic multiobjective optimization problems," Xitong Fangzhen Xuebao / Journal of System Simulation, vol. 23, no. 2, pp. 288-293, 2011.

[12] X. Li, J. Branke, and M. Kirley, "On performance metrics and particle swarm methods for dynamic multiobjective optimization problems," in Proceedings of the 2007 IEEE Congress on Evolutionary Computation, CEC 2007, pp. 576-583, September 2007.

[13] S. X. Yang and C. H. Li, "A clustering particle swarm optimizer for locating and tracking multiple optima in dynamic environments," IEEE Transactions on Evolutionary Computation, vol. 14, no. 6, pp. 959-974, 2010.

[14] M. Greeff and A. P. Engelbrecht, "Solving dynamic multiobjective problems with vector evaluated particle swarm optimisation," in Proceedings of the 2008 IEEE Congress on Evolutionary Computation, CEC 2008, pp. 2917-2924, June 2008.

[15] M. Helbig and A. Engelbrecht, "Influence of the archive size on the performance of the dynamic vector evaluated particle swarm optimisation algorithm solving dynamic multi-objective optimisation problems," in Proceedings of the IEEE Congress on Evolutionary Computation, CEC 2015, pp. 1926-1933, May 2015.

[16] J. Wei and L. Jia, "A novel particle swarm optimization algorithm with local search for dynamic constrained multi-objective optimization problems," in Proceedings of the 2013 IEEE Congress on Evolutionary Computation, CEC 2013, pp. 2436-2443, June 2013.

[17] M. Helbig and A. P. Engelbrecht, "Heterogeneous dynamic vector evaluated particle swarm optimisation for dynamic multi-objective optimisation," in Proceedings of the 2014 IEEE Congress on Evolutionary Computation, CEC 2014, pp. 3151-3159, July 2014.

[18] J. Wei and Y. Wang, "Hyper rectangle search based particle swarm algorithm for dynamic constrained multi-objective optimization problems," in Proceedings of the IEEE Congress on Evolutionary Computation, pp. 1-8, 2012.

[19] C. Hu, H. Yao, and X. Yan, "Multiple particle swarms coevolutionary algorithm for dynamic multi-objective optimization problems and its application," Journal of Computer Research and Development, vol. 50, no. 6, pp. 1313-1323, 2013.

[20] F. Pan, W. Li, and Q. Gao, Dynamic Multi-Objective Particle Swarm Optimizer Algorithm And Its Applications, Beijing Institute of Technology Press, Beijing, China, 2014.

[21] Y. He, H. Yu, B. Cheng, and D. Chen, "Multi-objective particle swarm optimization approach to solution of fed-batch bioreactor dynamic multi-objective optimization," Huagong Xuebao/Journal of Chemical Industry and Engineering, vol. 58, no. 5, pp. 1262-1270, 2007.

[22] $\mathrm{H} . \mathrm{Qu}$ and J. Wu, "The resource-constrained R\&G project scheduling problem based on dynamic multi-object particle swarm optimization algorithm," Systems Engineering, vol. 25, no. 9, pp. 98-102, 2007.

[23] M. Farina, K. Deb, and P. Amato, "Dynamic multiobjective optimization problems: test cases, approximations, and applications," IEEE Transactions on Evolutionary Computation, vol. 8, no. 5, pp. 425-442, 2004.

[24] A. Zhou, Y. Jin, and Q. Zhang, "A Population prediction strategy for evolutionary dynamic multiobjective optimization," IEEE Transactions on Cybernetics, vol. 44, no. 1, pp. 40-53, 2014. 
[25] D. Zhang, Digital Image Processing with MATLAB, China Machine Press, Beijing, China, 2012.

[26] J. Kennedy and R. Eberhart, "Particle swarm optimization," in Proceedings of the IEEE International Conference on Neural Networks, pp. 1942-1948, Perth, Australia, December 1995. 


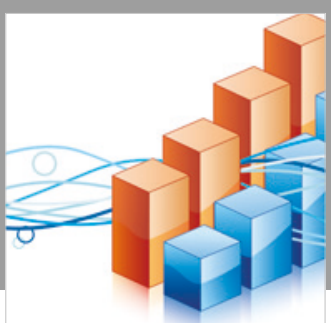

Advances in

Operations Research

\section{-n-m}
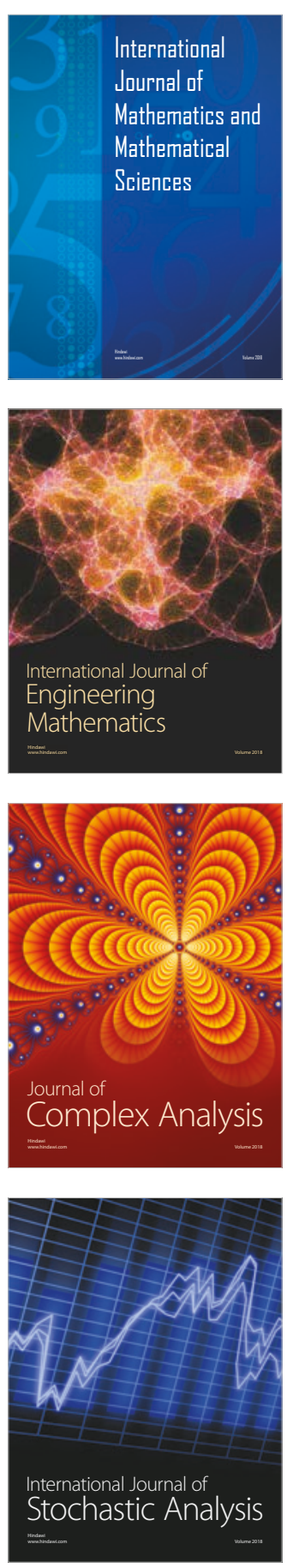
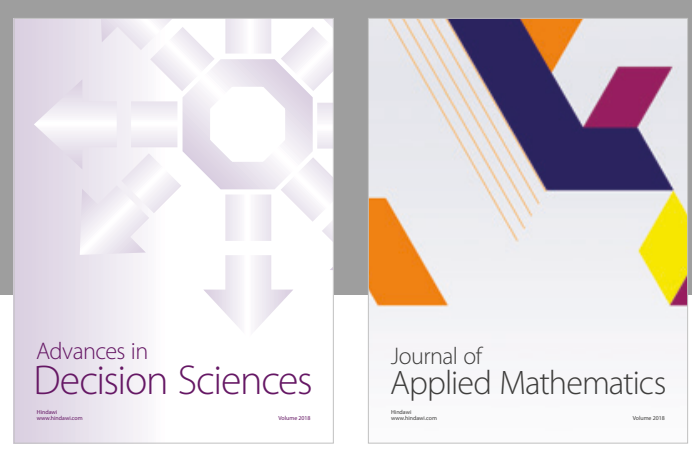

Journal of

Applied Mathematics
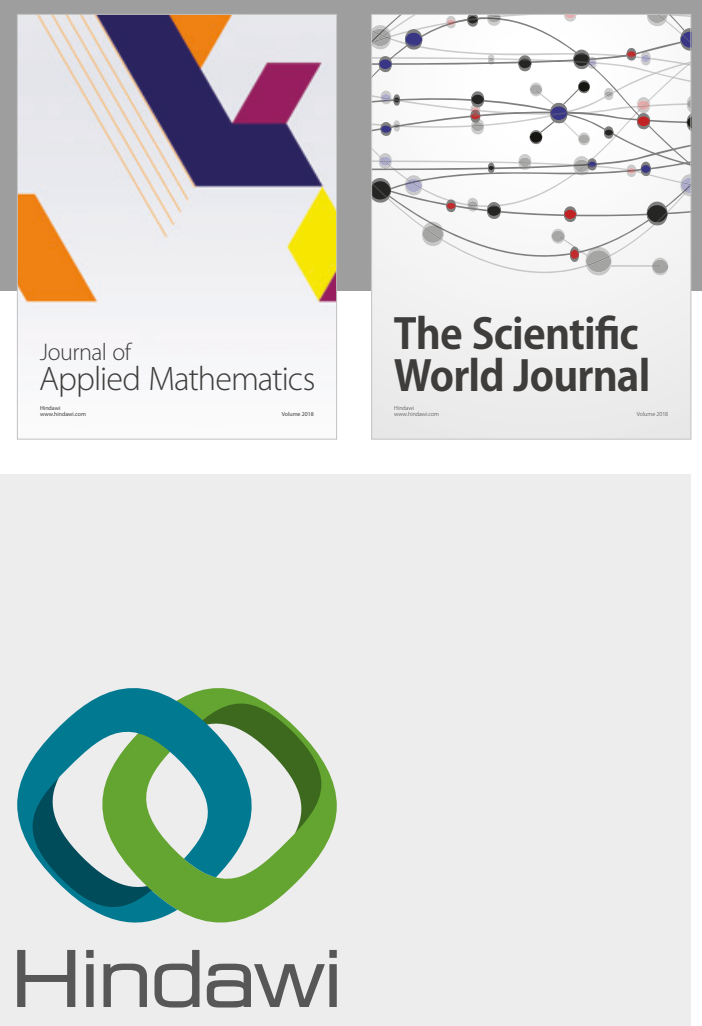

Submit your manuscripts at

www.hindawi.com

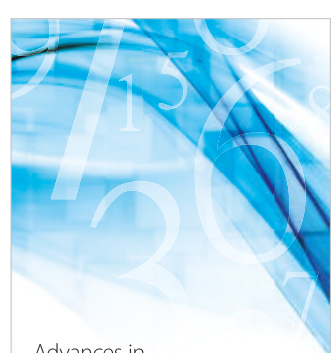

Advances in
Numerical Analysis
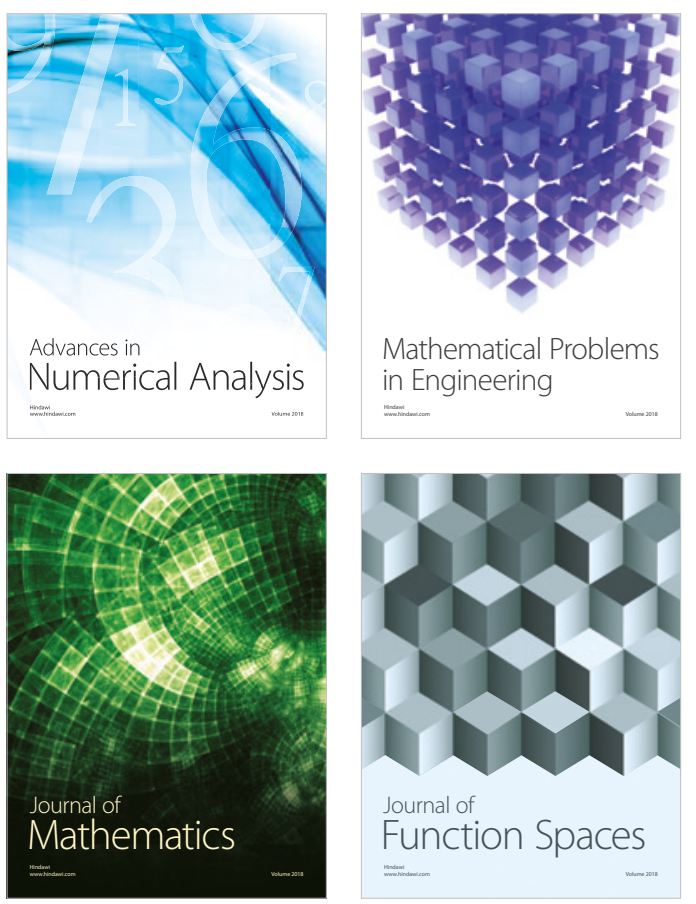

Mathematical Problems in Engineering

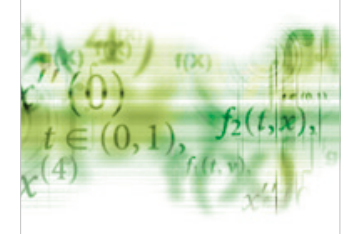

International Journal of

Differential Equations

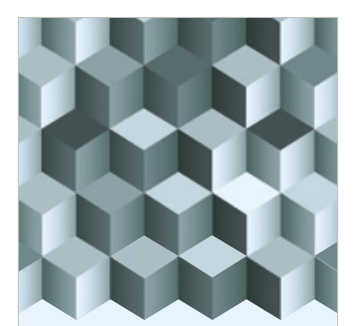

Journal of

Function Spaces

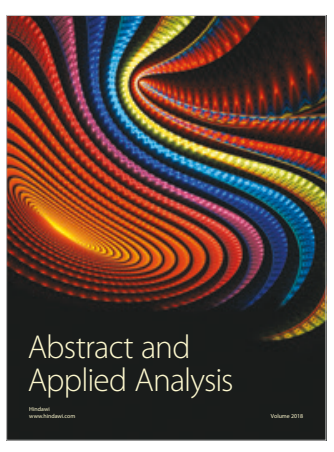

The Scientific

World Journal

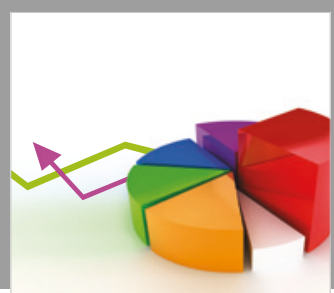

Journal of

Probability and Statistics
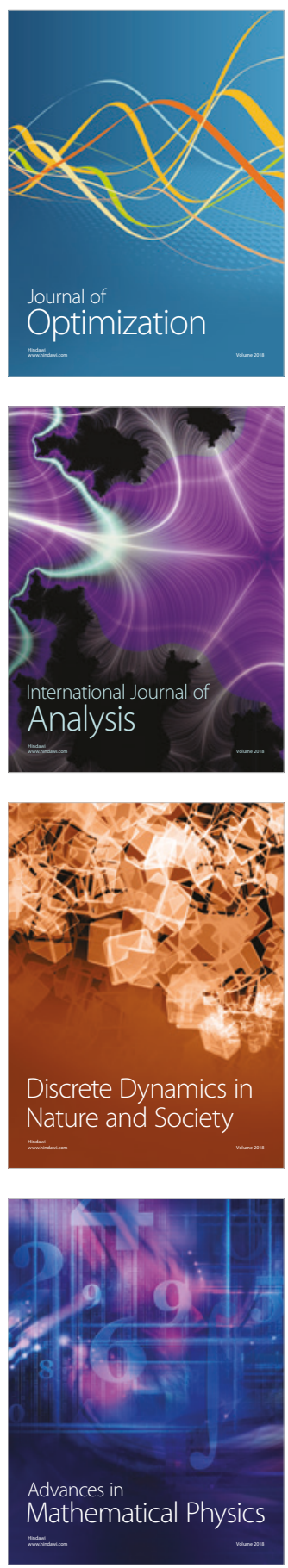